\title{
Review of the ${ }^{\text {nat }} C(n, \gamma)$ cross section and criticality calculations of the graphite moderated reactor BR1
}

\author{
C.J. Díez \\ A. Stankovskiy \\ E. Malambu \\ G. Žerovnik \\ P. Schillebeeckx' \\ G. Van den Eynde \\ J. Heyse O. Cabellos
}

\begin{abstract}
A B S T R A C T
A review of the experimental data for ${ }^{\text {nat }} \mathrm{C}(\mathrm{n}, \gamma)$ and ${ }^{12} \mathrm{C}(\mathrm{n}, \gamma)$ was made to identify the origin of the ${ }^{\text {nat }} \mathrm{C}$ capture cross sections included in evaluated data libraries and to clarify differences observed in neutronic calculations for graphite moderated reactors using different libraries. The performance of the JEFF-3.1.2 and ENDF/B-VII.1 libraries was verified by comparing results of criticality calculations with experimental results obtained for the BR1 reactor. This reactor is an air-cooled reactor with graphite as moderator and is located at the Belgian Nuclear Research Centre SCK•CEN in Mol (Belgium). The results of this study confirm conclusions drawn from neutronic calculations of the High Temperature Engineering Test Reactor (HTTR) in Japan. Furthermore, both BR1 and HTTR calculations support the capture cross section of ${ }^{12} \mathrm{C}$ at thermal energy which is recommended by Firestone and Révay. Additional criticality calculations were carried out in order to illustrate that the ${ }^{\text {nat }} \mathrm{C}$ thermal capture cross section is important for systems with a large amount of graphite. The present study shows that only the evaluation carried out for JENDL-4.0 reflects the current status of the experimental data.
\end{abstract}

\section{Introduction}

The results of criticality calculations for graphite moderated reactors are strongly influenced by the neutron induced capture reaction cross section on carbon that is used in the neutronic calculations. This has been demonstrated by the neutronic calculations done for the High Temperature Engineering Test Reactor (HTTR) in Japan, carried out by Goto et al. (2006, 2011). In Goto et al. (2011), criticality calculations for different numbers of loaded fuel columns of the HTTR core were performed using different cross section data libraries. Substantial differences were observed between the effective multiplication factor, $k_{\text {eff. }}$ The neutron capture cross section for carbon at thermal energy included in each library was pointed out as the main source of these differences. The calculated values for $k_{\text {eff }}$ were compared with experimental data obtained by Nojiri et al. (2004). The best agreement with experimental data was obtained using JENDL-4.0 (Shibata et al., 2011). In JENDL-4.0, the thermal capture cross section for carbon was changed to $3.86 \mathrm{mb}$ based on the recommendation made by Firestone et al. (2008). This value is $9 \%$ larger than the value in JENDL-3.3 (Shibata et al., 2002) and 15\% larger than those in ENDF/B-VII.0 (Chadwick et al., 2006) and JEFF-3.1.2 (Santamarina et al., 2009).

In this work, the performances of the JEFF-3.1.2 and the ENDF/ B-VII.1 (Chadwick et al., 2011) libraries were compared for the BR1 reactor, an air-cooled reactor with graphite as moderator located at the Belgian Nuclear Research Centre SCK•CEN in Mol (Belgium). Since we observed a rather large difference in $k_{\text {eff, }}$ we decided to perform an in-depth study in order to find out the origin of the discrepancy. It quickly became apparent that the choice of the evaluated cross section for graphite was the source of the difference and required more analysis.

To clarify the discrepancies between the results obtained using different evaluated nuclear data libraries, the experimental and recommended data for the thermal capture cross section of nat $\mathrm{C}$ and ${ }^{12} \mathrm{C}$ were reviewed. The results are summarised in Section 2. In Section 3, the results of the criticality calculations for the BR1 reactor are reported and compared with experimental data. Finally, we present results of additional criticality calculations for other graphite systems. 
Table 1

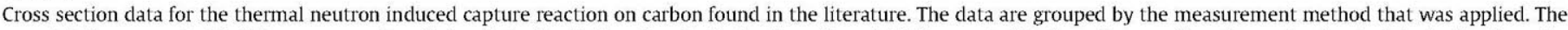

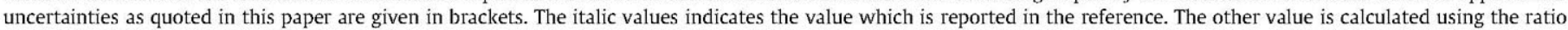
$\left.{ }^{\text {nat }} \mathrm{C}\left(\mathrm{n}_{t h}, \gamma\right)\right|^{12} \mathrm{C}\left(\mathrm{n}_{t h}, \gamma\right)=0.99345$

\begin{tabular}{|c|c|c|c|c|c|c|c|}
\hline \multirow{2}{*}{$\frac{\mathrm{ID}}{1}$} & \multirow{2}{*}{$\begin{array}{l}\text { Reference } \\
\text { Firestone and Révay (2011) }\end{array}$} & \multicolumn{2}{|c|}{${ }^{\text {nat }} \mathrm{C}\left(\mathrm{n}_{\text {th }}, \gamma\right) / \mathrm{mb}$} & \multicolumn{2}{|c|}{${ }^{12} \mathrm{C}\left(\mathrm{n}_{t h}, \gamma\right) / \mathrm{mb}$} & \multirow{2}{*}{$\begin{array}{l}\text { Method } \\
\text { Prompt } \gamma \text {-ray }\end{array}$} & \multirow{2}{*}{$\frac{\text { EXFOR entry }}{-}$} \\
\hline & & 3.875 & & 3.90 & $(0.06)$ & & \\
\hline 2 & Matsue and Yonezawa (2004) & 3.785 & & 3.81 & $(0.11)$ & Prompt $\gamma$-ray & - \\
\hline 3 & Jurney et al. (1982) & 3.507 & & 3.53 & $(0.07)$ & Prompt $\gamma$-ray & 12744.003 \\
\hline 4 & Prestwich et al. (1981) & 3.50 & $(0.16)$ & 3.523 & & Prompt $\gamma$-ray & 10997.002 \\
\hline 5 & Jurney and Motz (1963) & 3.775 & & 3.80 & $(0.40)$ & Prompt $\gamma$-ray & 11133.004 \\
\hline 6 & Nichols (1960) & 3.65 & $(0.15)$ & 3.674 & & Pile oscillator & 11290.006 \\
\hline 7 & Koechlin et al. (1957) & 3.85 & $(0.15)$ & 3.875 & & Pile oscillator & 22552.002 \\
\hline 8 & Muehlhause et al. (1957) & 3.50 & $(0.30)$ & 3.523 & & Pile oscillator & 11351.002 \\
\hline 9 & Sagot and Tellier (1963) & 3.72 & $(0.15)$ & 3.745 & & Pulsed neutrons & 22516.002 \\
\hline 10 & Starr and Price (1962) & 3.83 & $(0.06)$ & 3.855 & & Pulsed neutrons & 11272.002 \\
\hline 11 & Hendrie et al. (1958), Price (1964) & 3.44 & $(0.08)$ & 3.463 & & Diffusion length & 11278.002 \\
\hline 12 & Nichols (1960) & 3.57 & $(0.03)$ & 3.594 & & Reactivity & 11290.006 \\
\hline 13 & Henning (1950) & 3.278 & & 3.30 & $(0.15)$ & Mass spectrometry & - \\
\hline 14 & EXFOR data point & 3.80 & $(0.10)$ & 3.825 & & - & 22545.002 \\
\hline
\end{tabular}

\section{Experimental and evaluated data for neutron induced reactions in carbon}

In this section, experimental data for the neutron induced capture cross section of ${ }^{12} \mathrm{C}$ and ${ }^{\text {nat }} \mathrm{C}$ reported in the literature and/or stored in the EXFOR library (Otuka et al., 2011) are discussed. In addition, the ${ }^{\text {nat }} C(\mathrm{n}, \gamma)$ cross sections recommended in JEFF-3.1.2, JENDL-3.3, JENDL-4.0, ENDF/B-VI.1 and ENDF/B-VII.1 are reviewed and compared. The origin of these cross sections is verified in order to clarify the differences between them. The data are summarised in Tables 1-3 and represented in Figs. 1-3. The JANIS tool (Soppera et al., 2011) was used to retrieve the data from EXFOR and the evaluated data libraries.

\subsection{Experimental data}

\subsubsection{Thermal neutron induced capture cross section}

Experimental cross section data reported in the literature for the thermal neutron induced capture cross section of ${ }^{n a t} \mathrm{C}$ and ${ }^{12} \mathrm{C}$, ${ }^{\text {nat }} \mathrm{C}\left(\mathrm{n}_{t h}, \gamma\right)$ and ${ }^{12} \mathrm{C}\left(\mathrm{n}_{t h}, \gamma\right)$, are listed in Table 1 and shown in Fig. 1. The data are grouped by the measurement methodology. The list is based on a compilation of experimental data by Mughabghab (2003) and Firestone and Révay (2011). In Table 1, the values for both nat $C\left(n_{t h}, \gamma\right)$ and ${ }^{12} C\left(n_{t h}, \gamma\right)$ are given. To derive the value for ${ }^{\text {nat }} \mathrm{C}\left(\mathrm{n}_{t h}, \gamma\right)$ from ${ }^{12} \mathrm{C}\left(\mathrm{n}_{t h}, \gamma\right)$ (and vice versa), a ratio of ${ }^{\text {nat }} \mathrm{C}\left(\mathrm{n}_{t h}, \gamma\right) /{ }^{12} \mathrm{C}\left(\mathrm{n}_{t h}, \gamma\right)=0.99345 \pm 0.00050$ is used. This ratio is based on the representative isotopic composition of ${ }^{\text {nat }} \mathrm{C}$ given by Rosman and Taylor (1998) and the capture cross-section ratio ${ }^{13} \mathrm{C}\left(\mathrm{n}_{t h}, \gamma\right) /{ }^{12} \mathrm{C}\left(\mathrm{n}_{t h}, \gamma\right)=0.388 \pm 0.010$ determined by Mughabghab et al. (1982). The value for ID = 2 in Table 1 has been derived by Firestone and Révay (2011) from the partial capture cross section data reported by Matsue and Yonezawa (2004).

In Fig. 1 , the ${ }^{12} C\left(n_{t h}, \gamma\right)$ cross section value of $3.53 \pm 0.07 \mathrm{mb}$ suggested by Mughabghab (2003), Said (2006) and $3.84 \pm 0.06 \mathrm{mb}$ recommended by Firestone and Révay (2011) are also shown. As noted by Firestone and Révay (2011), the values in Table 1 fall into two groups. One group is consistent with the value suggested by Mughabghab (2003), Said (2006), which is based on the result obtained by Jurney et al. (1982). The other group is consistent with the value recommended by Firestone and Révay (2011), which is strongly influenced by their result obtained from prompt $\gamma$-ray measurements at the cold neutron beam of the Budapest Neutron Centre using a coaxial HPGe detector with Compton suppression (Firestone and Révay, 2011). Partial capture cross sections were measured with a variety of stoichiometric carbon compounds, all
Table 2

An overview of experiments performed to determine the capture cross section for ${ }^{12} \mathrm{C}(\mathrm{n}, \gamma)$ and ${ }^{\mathrm{nat}} \mathrm{C}(\mathrm{n}, \gamma)$ as a function of neutron energy.

\begin{tabular}{llll}
\hline Reference & Energy range (keV) & EXFOR entry & Isotope \\
\hline Kikuchi et al. (1998) & 550 & - & ${ }^{12} \mathrm{C}$ \\
Shima et al. (1995) & $12-250$ & 22345.003 & ${ }^{12} \mathrm{C}$ \\
Ohsaki et al. (1994) & $12-250$ & 23002.003 & ${ }^{12} \mathrm{C}$ \\
Nagai et al. (1991) & 30 & 22271.007 & ${ }^{12} \mathrm{C}$ \\
Gibbons et al. (1961) & 65 and 167 & 11329.003 & ${ }^{\text {nat } C}$ \\
\hline
\end{tabular}

of which yielded consistent results. In this way, the impact of systematic effects due to flux normalisation, sample properties and reference cross section was largely reduced. Since the nuclear level scheme of ${ }^{13} \mathrm{C}$ is well established and complete, the capture cross section at thermal energy can be derived from (Schillebeeckx et al., 2012b): (1) the sum of all partial cross sections of primary transitions depopulating the ground state, (2) the sum of the partial capture cross sections of the transitions that feed the ground state, or (3) the sum of all the partial cross sections weighted with the energy of the transition. Using the partial cross sections provided by Firestone and Révay (2011), the results obtained by applying the three methods were fully consistent.

\subsubsection{Neutron induced capture cross section as a function of neutron energy}

Experiments to study the capture cross section as a function of neutron energy are rather scarce. All ${ }^{\text {nat }} \mathrm{C}(\mathrm{n}, \gamma)$ and ${ }^{12} \mathrm{C}(\mathrm{n}, \gamma)$ cross section data stored in the EXFOR library are summarised in Table 2 and shown in Figs. 2 and 3. All these experiments have been performed with a modest energy resolution and are limited to a maximum energy of $250 \mathrm{keV}$. The cross section data provided by Shima et al. (1995), Ohsaki et al. (1994) and Nagai et al. (1991) for ${ }^{12} \mathrm{C}(\mathrm{n}, \gamma)$ are in good agreement with each other.

Table 3

A comparison of the total, elastic scattering and capture cross sections at thermal energy as included in ENDF/B-VI.1, ENDF/B-VII.1, JEFF-3.1.2, JENDL-3.3 and JENDL-4.0.

\begin{tabular}{|c|c|c|c|c|c|}
\hline $\begin{array}{l}\text { Cross-section } \\
\text { (barns) }\end{array}$ & $\begin{array}{l}\text { ENDF/B- } \\
\text { VI.1 }\end{array}$ & $\begin{array}{l}\text { ENDF/B- } \\
\text { VII.1 }\end{array}$ & $\begin{array}{l}\text { JEFF- } \\
3.1 .2\end{array}$ & $\begin{array}{l}\text { JENDL- } \\
3.3\end{array}$ & $\begin{array}{l}\text { JENDL- } \\
4.0\end{array}$ \\
\hline$\sigma\left(\mathrm{n}_{t h}, \mathrm{tot}\right)$ & 4.74260 & 4.74310 & 4.74260 & 4.74273 & 4.74306 \\
\hline$\sigma\left(\mathrm{n}_{t h}, \mathrm{n}\right)$ & 4.73924 & 4.73924 & 4.73924 & 4.73920 & 4.73920 \\
\hline$\sigma\left(\mathrm{n}_{t h}, \gamma\right)$ & 0.00336 & 0.00386 & 0.00336 & 0.00353 & 0.00386 \\
\hline
\end{tabular}




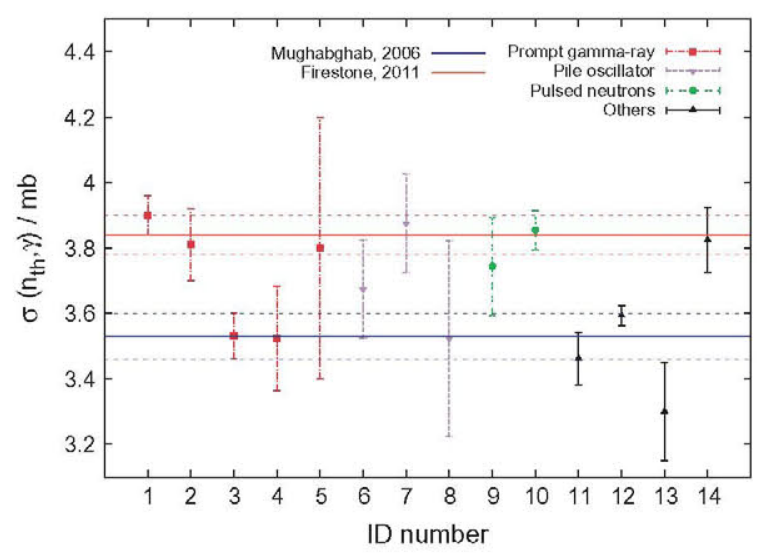

Fig. 1. Cross section data for the thermal neutron induced capture reaction on ${ }^{12} \mathrm{C}$ presented in Table 1 as a function of its ID number. The full lines represent the suggested values by Said (2006) and Firestone and Révay (2011). The dashed lines and the error bars represent the uncertainty within one standard deviation.

\subsection{Cross section data libraries}

The total, elastic scattering and capture cross sections at thermal energy included in the latest versions of the ENDF, JEFF and JENDL libraries, i.e. ENDF/B-VII.1, JEFF-3.1.2 and JENDL-4.0, together with those given in JENDL-3.3 and ENDF/B-VI.1 are listed in Table 3. The energy dependence of the capture cross sections in ENDF/B-VI.1 and JENDL-4.0, shown in Figs. 2 and 3, illustrates that below $1 \mathrm{keV}$ the capture cross section is supposed to be inversely proportional to the square root of the neutron energy (i.e. proportional to $\frac{1}{v}$, where $v$ is the neutron's velocity). This energy region is dominated by the contribution of s-wave neutrons with orbital angular momentum $l=0$. Hence, a different thermal capture cross section value will directly result in a difference between the cross sections in the region where the $\frac{1}{v}$ behaviour dominates.

The cross sections for incident neutron energy below $20 \mathrm{MeV}$ in ENDF/B-VII.1, JEFF-3.1.2 and JENDL-4.0 originate from two different evaluations. These are the evaluations performed for ENDF/BVI.1 and JENDL-4.0. The former is taken over in JEFF-3.1.2 and the latter in ENDF/B-VII.1.

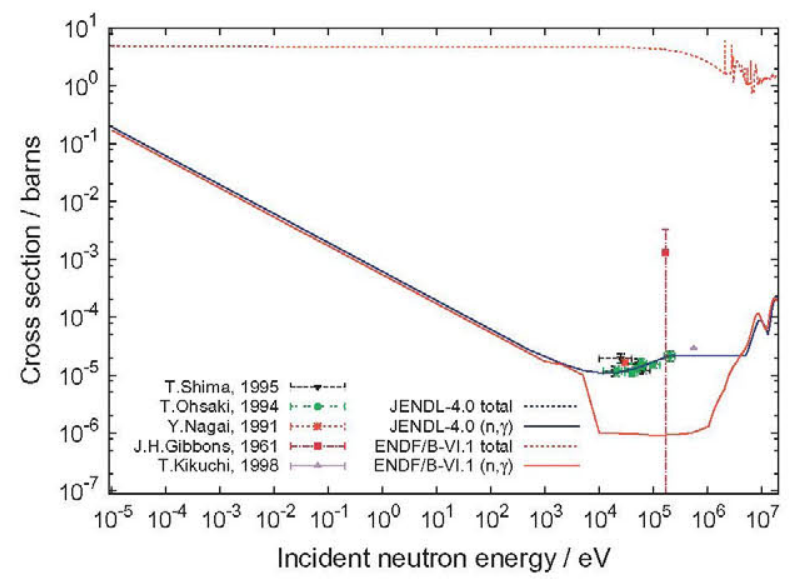

Fig. 2. A comparison of the total and capture cross section for ${ }^{\text {nat }} \mathrm{C}$ as given in ENDF/ B-VI.1 and JENDL-4.0 data libraries as a function of incident neutron energy. The experimental data presented in Table 2 are also shown, multiplying the experimental ${ }^{12} \mathrm{C}$ cross section data by the 0.99345 for converting to ${ }^{\text {nat }} \mathrm{C}$ cross section values. The total cross sections of both libraries are presented for comparison with the capture cross sections.

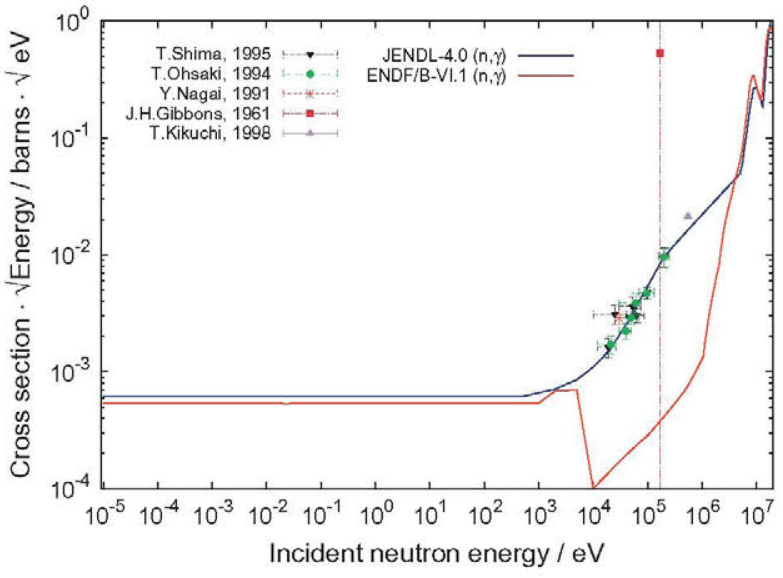

Fig. 3. The capture cross sections for ${ }^{\text {nat }} \mathrm{C}$ provided in the ENDF/B-VI.1 and JENDL4.0 libraries as a function of neutron energy. Experimental energy dependent capture cross sections reported in the EXFOR library and listed in Table 2, are also presented. Note that the cross sections are multiplied by the square root of the incident neutron energy and for the EXFOR data the ${ }^{12} \mathrm{C}$ cross section values are multiplied by a factor of 0.99345 for converting them into ${ }^{\text {nat }} \mathrm{C}$ cross section values.

\subsection{1. $E N D F / B-V I .1$}

The evaluation of the ${ }^{\text {nat }} C$ capture cross section in ENDF/B-VI.1 is based on the work of Fu (1990), Axton (1992), and is summarised by Rose and Fu (1991, p. 78-81). Two different regions are considered to evaluate the energy dependence:

- For incident neutron energies $E_{n}<1 \mathrm{keV}$, the cross section follows a $\frac{1}{v}$ dependence which is normalised to a thermal capture cross section of $3.36 \mathrm{mb}$.

- In the energy region between $1 \mathrm{MeV}$ and $20 \mathrm{MeV}$, the cross section is derived from inverse reaction data reported by Cook (1957).

The uncertainty below $1 \mathrm{keV}$ is $3 \%$. However, no reference or explanation is given related to the origin of the cross section $\sigma\left(\mathrm{n}_{t h}\right.$, $\gamma)=3.36 \mathrm{mb}$ at thermal energy. There is also no link between this value and the experimental data listed in Table 1 . In addition, in the region between $1 \mathrm{keV}$ and $1 \mathrm{MeV}$ the energy dependence is not defined and the values in the file appear unphysical.

\subsubsection{JENDL-4.0}

The energy dependence of the capture cross section in JENDL4.0 is divided in three regions:

- Below $100 \mathrm{eV}$, the cross section follows a $\frac{1}{v}$ dependence normalised to a thermal capture cross section of $\sigma\left(\mathrm{n}_{t h}, \gamma\right)=3.86 \mathrm{mb}$ based on (Firestone et al., 2008).

- Between $100 \mathrm{eV}$ and $5 \mathrm{MeV}$, reference is made in the description of the file to a private communication without any further explanation.

- In the region between $5 \mathrm{MeV}$ and $20 \mathrm{MeV}$, the cross section is derived from the inverse reaction data reported by Cook (1957).

The uncertainty on the capture cross section for neutron energies below $1 \mathrm{keV}$ is $5 \%$. The energy dependence in Fig. 2 shows that the experimental data listed in Table 2 (Shima et al., 1995; Nagai et al., 1991; Ohsaki et al., 1994) have been taken into account in the evaluation process to include the contribution of the components with an orbital angular momentum $l \geqslant 1$. The comparison of the capture cross sections in Fig. 3 reveals that only the JENDL-4.0 evaluation reflects the current status of the available experimental data. 


\section{Criticality calculations for the BR1 reactor}

\subsection{Description of the BR1 reactor}

The BR1 reactor (Wagemans et al., 2009) is an air-cooled, graphite moderated reactor operating on natural uranium fuel. Its first criticality was obtained in May 1956. It has been used and is currently used for neutron physics experiments, neutron irradiations, training of nuclear engineers and reactor operators and calibration of detectors.

The reactor is made up of a matrix of graphite blocks, with 829 channels as presented in Fig. 4. Of these channels, 552 are loaded with fuel, yielding a total load of about 25 tons of natural uranium. The fuel rods are made of metallic natural uranium contained in an aluminium cladding. The graphite present in the reactor has two different compositions: the graphite in the core ("Type A graphite") and the graphite of the reflector ("Type B graphite").

Its nominal thermal power is $4 \mathrm{MW}$ but since the 1970 s it has operated on a daily basis at a maximum power of $700 \mathrm{~kW}$ with a peak thermal flux of $3.5 \times 10^{11} \mathrm{n} /\left(\mathrm{cm}^{2} \mathrm{~s}\right)$. So-called "power-runs" at $1 \mathrm{MW}$ are possible but are limited to a couple of hours. Since the reactor has been operating at low power for most of its lifetime, the burn-up of the (still first core) fuel is very small (i.e. less than $0.3 \mathrm{MWd} / \mathrm{kg} \mathrm{U}$ ).

\subsection{BR1 reactor models}

For different applications in this reactor, detailed neutron flux calculations are required e.g. to complement activation measurements or to estimate irradiation times more accurately. In order to respond to these needs, a full model within the MCNPX-2.7e code (Pelowitz, 2008) has been developed for the reactor and validated against experimental results. In conjunction with the JEFF3.1.2 and ENDF/B-VII.1 libraries, this code is used as a reference neutronic analysis tool.

The aim of the present study is to assess which cross section library for ${ }^{\text {nat }} C$ produces a $k_{\text {eff }}$ value closest to unity using the full MCNP model for a critical configuration of the BR1 reactor. The main characteristics of the model for the BR1 reactor are summarised below.

- The used fuel is modelled calculating its elemental and isotopic composition from its first criticality up to now by using the ALEPH2 code (Stankovskiy and Van den Eynde, 2012) taking into account the power history of the reactor. However, due to the low operating power, the effect of the burn-up on $k_{\text {eff }}$ is negligible.
- No temperature distribution in the reactor is taken into account, so average values are used.

- The two different types of graphite, Type A and Type B, are modelled; however, we assume no variations of either densities or impurities between blocks of the same type (see Table 4).

- Impurities present in the graphite are modelled as an equivalent boron content. Due to the nature of the impurities present in the graphite (and their neutron absorption cross sections), their presence will have an important impact on the outcome of criticality calculations. Fortunately, thanks to characterisation experiments, the concentrations of these impurities are rather accurately know for Type B graphite, but that is not the case for Type A graphite. Simulations have shown that modelling only the impurities in graphite of Type B results in a difference

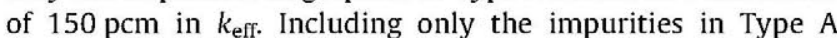
graphite provokes a difference of $950 \mathrm{pcm}$. The observed differences in $k_{\text {eff }}$, presented later in Tables 5 and 6 , remain the same even without modelling the impurities.

- The 18 control rods of BR1 are modelled. They consist of cadmium shell in an aluminium cladding with an active height of $1700 \mathrm{~mm}$.

Two different reactor states, Cold Zero Power (CZP) and Hot Full Power (HFP), are considered in this study. The characteristics of these states are described below.

\subsubsection{CZP state}

This state represents the start-up of the BR1 reactor when a subcritical approach is followed. In order to start up the reactor, the control rods are removed step-by-step to gradually reach criticality. The integral control rod worth is around $160 \mathrm{pcm}$ per rod on average. By analysing the count rate in neutron detectors and comparing these values to the values obtained in previous steps, the position of the control rods required to obtain criticality can be estimated. This process allows a safe approach to criticality without a risk of surpassing and hence producing a divergence of neutron flux and power.

When the reactor becomes critical, there is not yet enough fission power produced to induce any temperature increase in the system. The BR1 reactor produces about $200 \mathrm{~W}$ of thermal power at criticality after such a subcritical approach. As a result, the whole reactor can be considered in cold conditions with a well-defined constant temperature.

From the modelling point of view, this state is easy to model since the model does not need to take into account a temperature field and related consequences like Doppler broadening of U-238 resonances, a spectrum shift or thermal expansion of materials.

By putting the exact axial position of the control rods (typically an insertion of $190 \mathrm{~cm}$ ) from the reactor at the CZP state into the
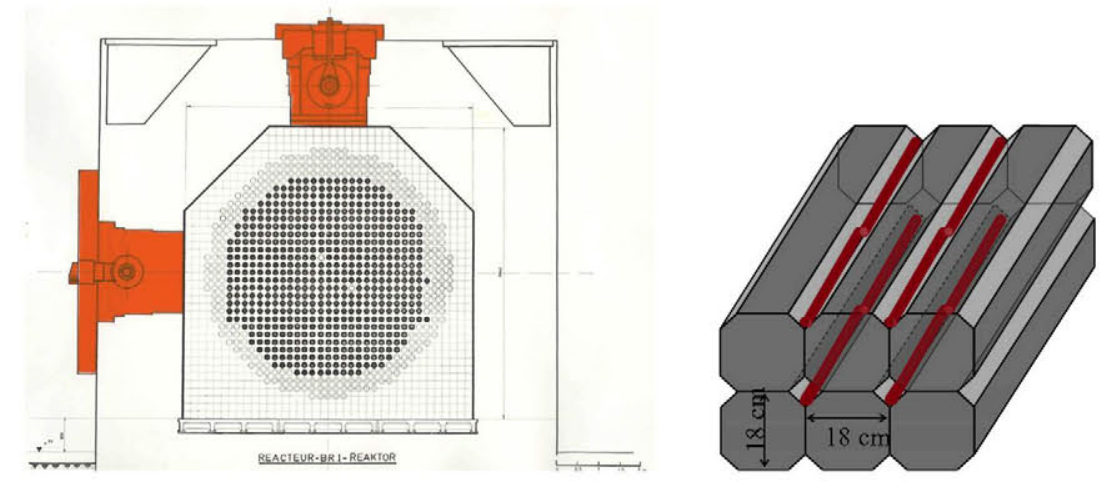

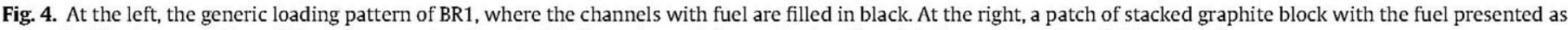
red rods. (For interpretation of the references to colour in this figure legend, the reader is referred to the web version of this article.) 
Table 4

Characteristics of the two different types of graphite implemented in the BR1 model for MCNP.

\begin{tabular}{|c|c|c|c|c|c|}
\hline & \multirow[b]{2}{*}{ Density $\left(\mathrm{g} / \mathrm{cm}^{3}\right)$} & \multicolumn{2}{|c|}{ Temperature } & \multirow{2}{*}{$\begin{array}{l}\text { Impurities } \\
\text { Boron equivalent (ppm) }\end{array}$} & \multirow[b]{2}{*}{ Observations } \\
\hline & & $\mathrm{CZP}(\mathrm{K})$ & $\operatorname{HFP}(\mathrm{K})$ & & \\
\hline Type A graphite & 1.7233 & 300 & 350 & 0.50 & Inner core - moderator \\
\hline Type B graphite & 1.6212 & 300 & 300 & 1.60 & Peripheral - reflector \\
\hline
\end{tabular}

model, we have a model that should be as close as possible to the reality of the reactor at this state. Comparing the calculated value of $k_{\text {eff }}$ to the reference experimental value $k_{\text {eff }}=1.00000$, we can assess the quality of the model and nuclear data files used.

\subsubsection{HFP state}

This state represents the $B R 1$ reactor working at $700 \mathrm{~kW}$ thermal power. The control rod position is typically $150 \mathrm{~cm}$ insertion at this state. To create a reference configuration for the calculation model, the control rod height is fixed at the same height as in the experiment and the equivalent boron concentration in the Type $A$ graphite is adjusted to reach a $k_{\text {eff }}$ value very close to unity in the simulation. This allows us to mimic the critical system without taking much trouble in exactly modelling the temperature field and its consequences.

The original equivalent content of boron in the Type A graphite was $0.38 \mathrm{ppm}$. In order to reach a value for $k_{\text {eff }}$ very close to unity this value was tuned to $0.50 \mathrm{ppm}$ when using the ENDF/B-VII. $1 \mathrm{li}$ brary. Using this value of impurities within the CZP state model, a $k_{\text {eff }}$ close to unity is still obtained ( $k_{\text {eff }}=0.99975 \pm 0.00004$ ).

\subsection{BR1 criticality calculations and results}

We have run calculations mainly with two libraries, JEFF-3.1.2 and ENDF/B-VII.1, and a modified version of the former. In this modified version, the thermal cross section of the $(n, \gamma)$ reaction is replaced by the one from the ENDF/B-VII.1 library because we expect only the $\frac{1}{v}$ component of the nat $C$ file to be of importance. In order not to perturb the original JEFF-3.1.2 file too much, we used the same interpolation rule and number of interpolation points as in the original file. We assumed the cross-over point to be the point where the original JEFF-3.1.2 and ENDF/B-VII.1 $(n, \gamma)$ cross sections intersect, i.e. $\sigma=16.37633 \mu \mathrm{b}$ at $E=1.4056 \mathrm{keV}$. The changes done in the JEFF-3.1.2 file of ${ }^{\text {nat }} \mathrm{C}$ are indicated in Fig. 5.

\subsubsection{BR1 pin-cell model}

Before tackling the full BR1 model, first a pin-cell model has been analysed. In this model, a single fuel channel of the BR1 reactor is modelled in an infinite lattice with a repeat pitch of $18 \mathrm{~cm}$. By modifying the pitch of the pin-cell, one can analyse the effect of the fuel to moderator ratio. The pitch value is varied from $8 \mathrm{~cm}$ to $36 \mathrm{~cm}$ taking steps of $2 \mathrm{~cm}$. The ${ }^{\text {nat }} \mathrm{U} /$ graphite weight ratio is calculated for all the cases.

Three sets of calculations have been performed: (i) using JEFF3.1.2 for all nuclei (as the reference case), (ii) using the ENDF/BVII.1 file for ${ }^{\text {nat }} \mathrm{C}$, and (iii) using our modified JEFF-3.1.2 file for ${ }^{\text {nat }} \mathrm{C}$. The results are presented in Fig. 6 . They show that the differences between the results obtained using different libraries increase with decreasing ${ }^{\text {nat }} \mathrm{U} / \mathrm{graphite}$ weight ratio. It is important to note that the difference obtained for the real value of the BR1 pin-cell pitch is $-1011 \mathrm{pcm}$ when using ENDF/B-VII.1 ( $-992 \mathrm{pcm}$ when using the modified JEFF-3.1.2 file).

\subsubsection{Full BR1 reactor model}

As a first step, we performed some perturbation calculations for the BR1 reactor model at HFP using MCNPX-2.7e by applying PERT cards. This technique allows the user to estimate changes in tallies or $k_{\text {eff }}$ values as a result of user-defined perturbations. These perturbations can be in cell material density, cell composition or cross section data. Multiple perturbations can be defined and analysed within the same run, reducing the calculation time needed.

For our perturbation analysis, we used the JEFF-3.1.2 cross section library as a reference and applied two perturbations:

Table 5

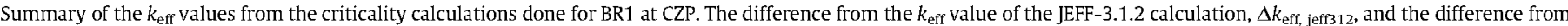
$k_{\text {eff }}=1.00000, \Delta k_{\text {eff, exp, }}$ is also given.

\begin{tabular}{|c|c|c|c|c|c|c|}
\hline Case & Base file & ${ }^{\mathrm{nat}} \mathrm{C}(\mathrm{n}, \gamma)\left(E_{\mathrm{n}}<1 \mathrm{keV}\right)$ & ${ }^{\mathrm{nat}} \mathrm{C}(\mathrm{n}, \gamma)\left(E_{n} \geqslant 1 \mathrm{keV}\right)$ & $k_{\text {eff }}$ & $\Delta k_{\text {eff, jeff312 }}(\mathrm{pcm})$ & $\Delta k_{\text {eff, } \exp }(\mathrm{pcm})$ \\
\hline CZP-1 & JEFF-3.1.2 & JEFF-3.1.2 & JEFF-3.1.2 & $1.00904 \pm 0.00005$ & - & +904 \\
\hline $\mathrm{CZP}-2$ & JEFF-3.1.2 & ENDF/B-VII.1 & ENDF/B-VII.1 & $0.99791 \pm 0.00004$ & $-\mathbf{1 1 0 3}$ & -209 \\
\hline CZP-3 & JEFF-3.1.2 & JEFF-3.1.2 modified & JEFF-3.1.2 & $0.99798 \pm 0.00004$ & -1096 & -202 \\
\hline CZP-4 & ENDF/B-VII.1 & ENDF/B-VII.1 & ENDF/B-VII.1 & $0.99857 \pm 0.00004$ & -1038 & -143 \\
\hline CZP-5 & ENDF/B-VII.1 & JEFF-3.1.2 & JEFF-3.1.2 & $1.00977 \pm 0.00004$ & +72 & +977 \\
\hline CZP-6 & ENDF/B-VII.1 & JEFF-3.1.2 modified & JEFF-3.1.2 & $0.99874 \pm 0.00004$ & -1021 & -126 \\
\hline
\end{tabular}

Table 6

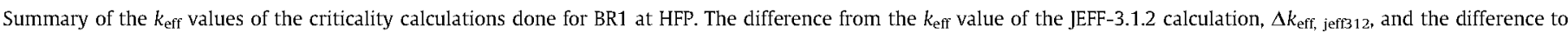
$k_{\mathrm{eff}}=1.00000, \Delta k_{\mathrm{eff}}$ exp, is also given.

\begin{tabular}{|c|c|c|c|c|c|c|}
\hline Case & Base file & ${ }^{\text {nat }} \mathrm{C}(\mathrm{n}, \gamma)\left(E_{n}<1 \mathrm{keV}\right)$ & ${ }^{\text {nat }} \mathrm{C}(\mathrm{n}, \gamma)\left(E_{n} \geqslant 1 \mathrm{keV}\right)$ & $k_{\mathrm{eff}}$ & $\Delta k_{\text {eff, jeff312 }}(\mathrm{pcm})$ & $\Delta k_{\text {eff, } \exp }(\mathrm{pcm})$ \\
\hline HFP-1 & JEFF-3.1.2 & JEFF-3.1.2 & JEFF-3.1.2 & $1.00999 \pm 0.00004$ & - & +999 \\
\hline HFP-2 & JEFF-3.1.2 & ENDF/B-VII.1 & ENDF/B-VII.1 & $0.99862 \pm 0.00004$ & $-\mathbf{1 1 2 6}$ & -138 \\
\hline HFP-3 & JEFF-3.1.2 & JEFF-3.1.2 modified & JEFF-3.1.2 & $0.99880 \pm 0.00004$ & -1108 & -120 \\
\hline HFP-4 & ENDF/B-VII. 1 & ENDF/B-VII.1 & ENDF/B-VII. 1 & $0.99944 \pm 0.00004$ & -1045 & -56 \\
\hline HFP-5 & ENDF/B-VII.1 & JEFF-3.1.2 & JEFF-3.1.2 & $1.01066 \pm 0.00004$ & +66 & +1066 \\
\hline HFP-6 & ENDF/B-VII.1 & JEFF-3.1.2 modified & JEFF-3.1.2 & $0.99958 \pm 0.00004$ & -1031 & -42 \\
\hline
\end{tabular}




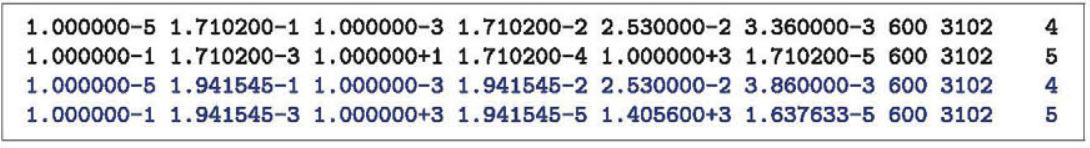

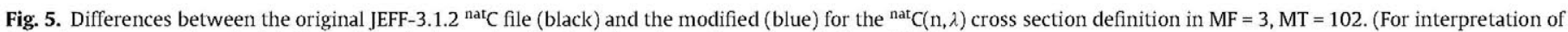
the references to colour in this figure legend, the reader is referred to the web version of this article.)

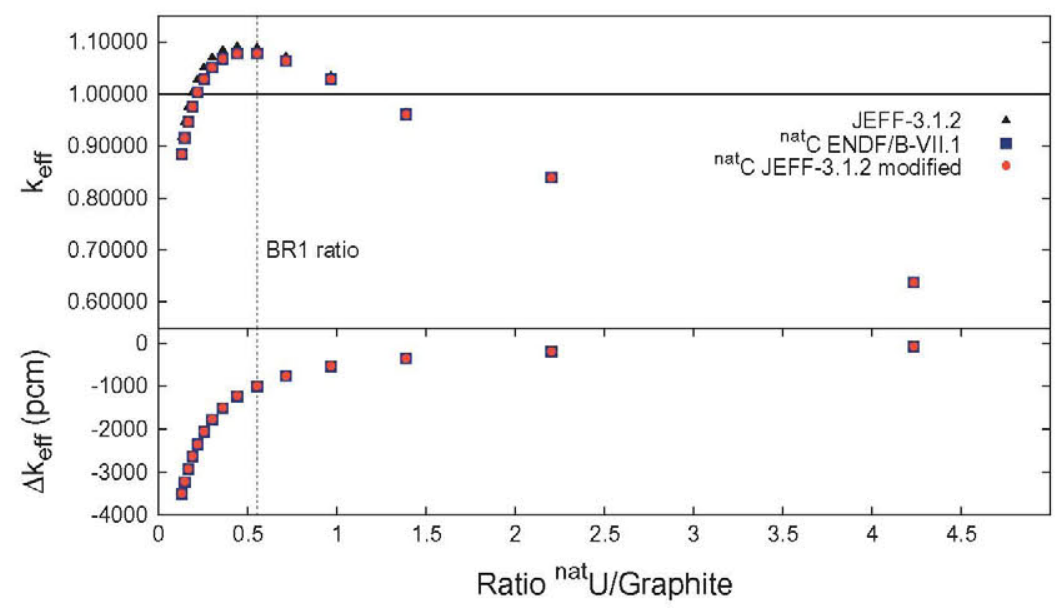

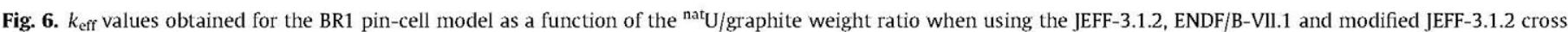

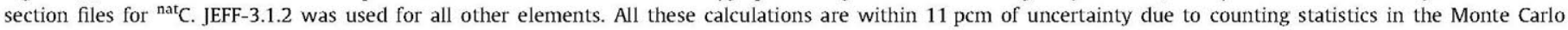
calculations.

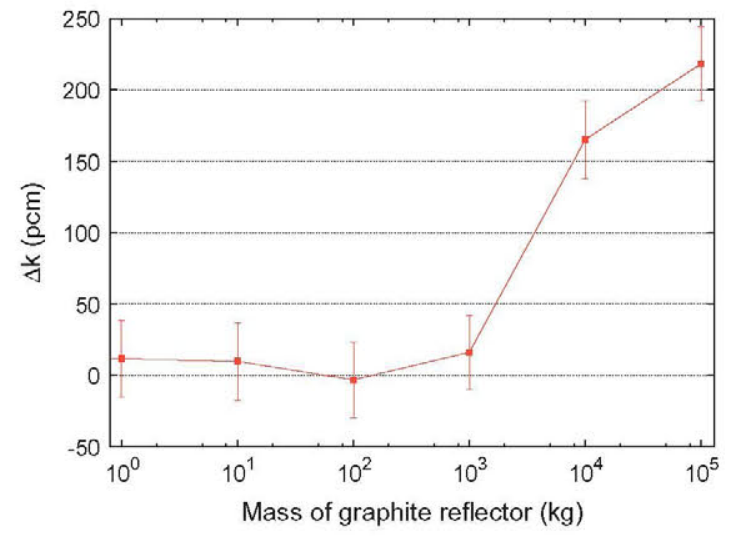

Fig. 7. Relative difference in $k_{\text {eff }}$ between the results using the cross section library of ${ }^{\text {nat }} \mathrm{C}$ from ENDF/B-VII.1 (reference) and JEFF-3.1.2, for a critical sphere of uranium (pure ${ }^{235} \mathrm{U}$ ) carbide with a diameter of $21.7 \mathrm{~cm}$ surrounded by a solid ${ }^{\text {nat }} \mathrm{C}$ external reflector sphere. For all other nuclei the ENDF/B-VII.1 library was used. The difference is given as a function of the amount of nat $\mathrm{C}$ in the reflector sphere.

1. Increase of the $(n, \gamma)$ cross section by $14.88 \%$ in the energy range from $10^{-5} \mathrm{eV}$ to $1 \mathrm{keV}$; this is equivalent to changing the normalisation point of the $\frac{1}{v}$ component from $3.36 \mathrm{mb}$ (JEFF-3.1.2) to $3.86 \mathrm{mb}$ (ENDF/B-VII.1).

2. Increase of the (n, $\gamma$ ) cross section by $150 \%$ in the energy range from $50 \mathrm{keV}$ to $1 \mathrm{MeV}$.

These two perturbations allow us to analyse the sensitivity of $k_{\text {eff }}$ to the ${ }^{\text {nat }} \mathrm{C}$ cross section in two different energy regions.

The result for the first perturbation shows a difference of $\Delta k_{\text {eff }}=-1211$ pcm for a $k_{\text {eff }}=1.01227 \pm 0.00010$. For the second perturbation, no variation of the $k_{\text {eff }}$ value is obtained. This shows that the differences between results obtained for the BR1 criticality calculations using the ${ }^{n a t} \mathrm{C}(\mathrm{n}, \gamma)$ cross section included in ENDF/B-VII.1 and JEFF-3.1.2 are only sensitive to the thermal region, despite the large difference introduced into the cross section files between $5 \mathrm{keV}$ and $5 \mathrm{MeV}$.

In order to eliminate the possible effect of the thermal scattering library $S(\alpha, \beta)$, two additional calculations are performed. Using the JEFF-3.1.2 library for all nuclei, the difference in $k_{\text {eff }}$ between using the thermal scattering library for graphite from JEFF-3.1.2 and ENDF/B-VII.1 is found to be negligible, i.e. not larger than $45 \mathrm{pcm}$.

Finally, six criticality calculations are carried out for CZP (results in Table 5) and HFP (results in Table 6) in order to show the effect of changing the ${ }^{\text {nat }} C(n, \gamma)$ cross section library. All the calculations presented above use the same thermal scattering library for graphite.

The results presented in Tables 5 and 6 clearly show that the thermal capture cross section value for ${ }^{\text {nat }} \mathrm{C}$ has an impact of above $1000 \mathrm{pcm}$ on the $k_{\text {eff }}$ calculations for a reactor such as BR1. They also demonstrate that there is a better agreement between experiment and calculations when using the (n, $\gamma$ ) cross section for ${ }^{\text {nat }} \mathrm{C}$ present in ENDF/B-VII.1 compared to JEFF-3.1.2.

\section{Benchmark studies}

To validate the importance of the thermal capture cross-section of nat $\mathrm{C}$ in graphite-moderated reactors, some benchmark studies are reviewed and performed.

In contrast to our experience with BR1 criticality calculations, calculations (Snoj et al., 2012a) for the TRIGA reactor at the Jožef Stefan Institute showed little impact on the $k_{\text {eff }}$ value when changing the ${ }^{\text {nat }} \mathrm{C}$ cross section from the one included in JEFF-3.1.2 to the one included in ENDF/B-VII.1. Since this reactor uses graphite only as reflector and not as core moderator, the fuel/graphite weight ratio is very high. It became apparent that this ratio would play a strong role in the sensitivity to the ${ }^{\text {nat }} \mathrm{C}$ cross section. Since the diffusion length is larger than the dimensions of the graphite reflector, the probability of the neutrons entering the reflector to be 


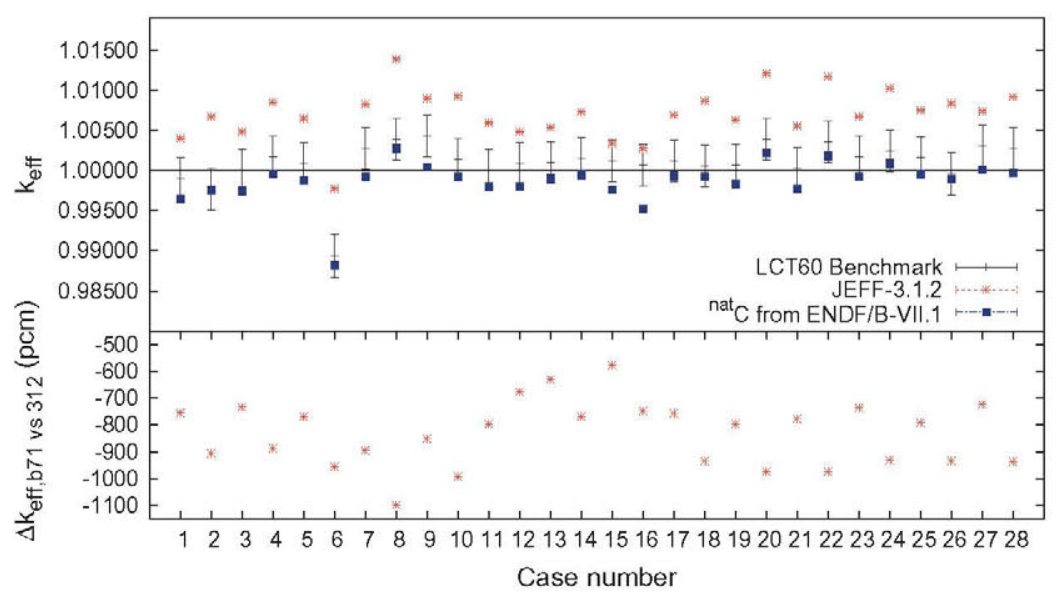

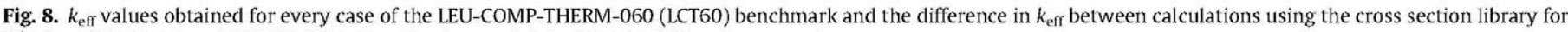
${ }^{\text {nat }} \mathrm{C}$ from JEFF-3.1.2 and ENDF/B-VII.1. For all other nuclei the JEFF-3.1.2 library was used.

absorbed in graphite is relatively small; most of them are either reflected back to the core or escape from the system. Furthermore, the fraction of neutrons entering the reflector is limited; it is significantly lower compared to the fraction of neutrons in contact with graphite in graphite moderated reactors. Therefore, we expect the sensitivity of the multiplication factor in water-moderated graphite-reflected reactors like TRIGA on the carbon thermal capture cross section to be small as corroborated by the calculations cited above, where the change in $k_{\text {eff }}$ when substituting the ${ }^{\text {nat }} \mathrm{C}$ cross section file from JEFF-3.1 to ENDF/B-VII.1 is within the $16 \mathrm{pcm}$ uncertainty due to counting statistics in the Monte Carlo calculations.

A criticality calculation for a sphere of uranium carbide with a critical diameter of $21.7 \mathrm{~cm}$ surrounded by a sphere of ${ }^{\text {nat }} \mathrm{C}$ was performed. The criticality calculations were done using the ENDF/B-VII.1 cross section library and with a varying amount of ${ }^{\text {nat }} \mathrm{C}$ in the external sphere. These calculations were repeated using the JEFF-3.1.2 cross section library for ${ }^{\text {nat }} C$; for all other nuclei the ENDF/B-VII.1 library was used. The difference in $k_{\text {eff }}$ due to this change of library in ${ }^{\text {nat }} \mathrm{C}$ was calculated as a function of the amount of ${ }^{\text {nat }} \mathrm{C}$ in the external sphere. The results, presented in Fig. 7, indicate that the difference becomes substantial when the mass of the ${ }^{\text {nat }} \mathrm{C}$ sphere is of the order of several tons, which is the case for BR1, but not for the TRIGA reactor at JSI.

Next, a first benchmark from the ICSBEP Handbook (NEA Nuclear Safety Committee, 2010), HEU-COMP-THERM-002 (HCTO2) was analysed looking at the effect of changing the ${ }^{\text {nat }} \mathrm{C}$ cross section library from JEFF-3.1.2 to ENDF/B-VII.1. In this benchmark experimental data are available for an NRX-A type fuel element which houses pyrolytic-graphite coated uranium-dicarbide spherical beds in a graphite matrix. These fuel elements were then placed in a water environment until criticality was reached. Since the amount of graphite is relatively small, differences smaller than $150 \mathrm{pcm}$ in $k_{\text {eff }}$ were observed when comparing results obtained with the different libraries.

As a second benchmark from the ICSBEP Handbook, the LEUCOMP-THERM-060 (LCT60) benchmark was selected. It is based on an experimental RBMK reactor (a uranium-graphite reactor) which has a large amount of nat $C$ in the form of graphite. Clearly, one expects that the differences due to switching the ${ }^{\text {nat }} \mathrm{C}$ cross section library from JEFF-3.1.2 to ENDF/B-VII.1 will be larger than for the HCT02 benchmark. Fig. 8 proves this as the differences in $k_{\text {eff }}$ range from $-600 \mathrm{pcm}$ to $-1100 \mathrm{pcm}$.

\section{Conclusions}

Because of the discrepancies found between the calculated values for $k_{\text {eff }}$ using the reference MCNP/X model of the BR1 reactor using JEFF-3.1.2 and ENDF/B-VII.1, we performed an in-depth analysis to pinpoint the origin of these differences. This analysis showed that the $(n, \gamma)$ cross section from the ${ }^{\text {nat }} \mathrm{C}$ library file was the culprit.

Detailed analysis of the ${ }^{\text {nat }} \mathrm{C}$ library files revealed several inconsistencies in the cross section files themselves. Two serious issues were identified in the JEFF-3.1.2 file: a different and not referenced normalisation value for the $\frac{1}{v}$ law applied in the thermal region and a non-physical behaviour for the epithermal region.

Neutronic calculations for different models of the BR1 reactor were carried out. By using the PERT card approach in $\mathrm{MCNP} / \mathrm{X}$, it was shown that the difference in normalisation for the $\frac{1}{v}$ law in the thermal neutron energy region was solely responsible for the difference between the libraries. The anomalous behaviour of the JEFF-3.1.2 cross section for the epithermal region had no influence at all.

For the BR1 pin-cell model, a difference of about $1000 \mathrm{pcm}$ is obtained between results using the JEFF-3.1.2 and ENDF/B-VII.1 or modified JEFF-3.1.2 files for ${ }^{\text {nat }} \mathrm{C}$ for the case where the fuel to graphite weight ratio is representative of the BR1 situation. It shows that for small values of the ${ }^{\text {nat }} \mathrm{U} /$ graphite weight ratio for graphite-moderated reactors the differences between results obtained using different values for the ${ }^{\text {nat }} \mathrm{C}$ thermal capture cross section are important.

For the full model of BR1 at Cold Zero Power and Hot Full Power, again a difference of about $1000 \mathrm{pcm}$ is observed between results obtained with ENDF/B-VII.1 and JEFF-3.1.2. As presented before, these differences are mainly due to the part of capture cross section that is dominated by the $\frac{1}{v}$ component, which is normalised to the cross section at thermal neutron energy.

For both states (CZP and HFP), there is a good agreement between the experimental result and the result of calculations using the cross section data in ENDF/B-VII.1. Since the cross section data for ${ }^{\text {nat }} \mathrm{C}$ in ENDF/B-VII.1 are taken from JENDL-4.0, this confirms previous results reported for HTTR. Hence, it provides an additional argument for a re-evaluation of the capture cross section of ${ }^{12} \mathrm{C}$ in the JEFF-3.1.2 library taking into account the value $\sigma\left(n_{t h}, \gamma\right)=$ $3.84 \pm 0.06 \mathrm{mb}$ recommended by Firestone and Révay (2011) for ${ }^{12} \mathrm{C}$. 
Finally, using the two cross section libraries JEFF-3.1.2 and ENDF/B-VII.1, it was shown that a certain minimum amount of graphite mass is needed to have a pronounced influence from the difference in cross section library for ${ }^{\text {nat }} \mathrm{C}$. This has been illustrated by a number of benchmark studies of graphite systems. This minimum amount is reached typically for reactors that use graphite both as core moderator and as reflector material, as the BR1 reactor at SCK•CEN.

\section{Acknowledgements}

The first author would like to thank SCK•CEN and EC-JRC-IRMM for hosting him during his internship in 2012, and for providing him all the materials and resources needed for performing this work.

The research leading to these results has received funding from the European Atomic Energy Community's Seventh Framework Programme [FP7/2007-2013] under Grant Agreement Nr. 249671, (FP7-EURATOM-FISSION-2009:Project ANDES/249671). This work is also partially supported by the Ministry of Education of Spain with the FPU Program for teaching and researching formation under Grant AP2009-1801 for the first author.

\section{References}

Axton, E.J. 1992. Tech. Rep. NISTIR 4838, ENDF-353.

Chadwick, M.B., Obložinský, P., Herman, M., Greene, N.M., McKnight, R.D., Smith, D.L. Young, P.G., MacFarlane, R.E., Hale, G.M., Frankle, S.C., Kahler, A.C., Kawano, T., Little, R.C., Madland, D.G., Moller, P., Mosteller, R.D., Page, P.R., Talou, P., Trellue, H., White, M.C., Wilson, W.B., Arcilla, R., Dunford, C.I., Mughabghab, S.F. Pritychenko, B., Rochman, D., Sonzogni, A.A., Lubitz, C.R., Trumbull, T.H. Weinman, J.P., Brown, D.A., Cullen, D.E., Heinrichs, D.P., McNabb, D.P., Derrien, H., Dunn, M.E., Larson, N.M., Leal, L.C., Carlson, A.D., Block, R.C., Briggs, J.B., Cheng E.T., Huria, H.C., Zerkle, M.L., Kozier, K.S., Courcelle, A., Pronyaev, V., van der Marck, S.C., 2006. ENDF/B-VII.0: next generation evaluated nuclear data library for nuclear science and technology. Nuclear Data Sheets 107 (12), 2931-3060.

Chadwick M.B., Herman M., Obložinský, Dunn M.E., Danon, Y., Kahler, A.C., Smith D.L., Pritychenko, B., Arbanas, G., Arcilla, R., Brewer, R., Brown, D.A., Capote, R., Carlson, A.D., Cho, Y.S., Derrien, H., Guber, K., Hale, G.M., Hoblit, S., Holloway, S. Johnson, T.D., Kawano, T., Kiedrowski, B.C., Kim, H., Kunieda, S., Larson, N.M., Leal, L. Lestone, J.P., Little, R.C., McCutchan, E.A., MacFarlane, R.E., Macinnes, M., Mattoon, C.M., McKnight, R.D., Mughabghab, S.F., Nobre, G.P.A., Palmiotti, G., Palumbo, A., Pigni, M.T., Pronyaev, V.G., Sayer, R.O., Sonzogni, A.A., Summers, N.C., Talou, P., Thompson, I.J., Trkov, A., Vogt, R.L., van S.C., der Marck, Wallner, A., White, M.C., Wiarda, D., Young, P.G., 2011. ENDF/B-VII.1 Nuclear data for science and technology: cross sections, covariances, fission product yields and decay data. Nuclear Data Sheets 112 (12), 2887-2996.

Cook, Barnett C. 1957. Photodisintegration of ${ }^{13}$ C. Physical Review 106, 300-314.

EXFOR database, 1956.http://www-nds.iaea.org/exfor/servlet/X4sGetInfo?subID= 22545002 \&pointer $=$

Firestone, R.B., Révay, Z., 2011. Comparison of IUPAC $k_{0}$ values and neutron cross sections to determine a self-consistent set of data for neutron activation analysis. Proceedings in Radiochemistry, 305-312.

Firestone, R.B., Krtička, M., McNabb, D.P., Sleaford, B., Agvaanluvsan, U., Belgya, T. Revay, Zs., 2008. New methods for the determination of total radiative thermal neutron capture cross sections. In: AIP Conference Proceedings. Vol. 1005. pp. 26-29.

Fu, C.Y., 1990. Evaluated cross sections for neutron scattering from natural carbon below $2 \mathrm{MeV}$ including $\mathrm{R}$ matrix fits to ${ }^{13} \mathrm{C}$ resonances. Nuclear Science and Engineering 106 (4), 494-500.

Gibbons, J.H., MacKlin, R.L., Miller, P.D., Neiler, J.H., 1961. Average radiative capture cross sections for 7- to 170-keV neutrons. Physical Review 122 (1), 182-201.

Goto, M., Nojiri, N., Shimakawa, S., 2006. Neutronics calculations of HTTR with several nucl ear data libraries. Journal of Nuclear Science and Technology 43 (10), 1237-1244.

Goto, M., Shimakawa, S., Nakao, Y., 2011. Impact of revised thermal neutron capture cross section of carbon stored in JENDL- 4.0 on HTTR criticality calculation. Journal of Nuclear Science and Technology 48 (7), 965-969.

Hendrie, J.M., Phelps, J.P., Price, G.A., Weinstock, E.V. 1958. Slowing down and diffusion lengths of neutrons in graphite-bismuth systems. In: Second Internat. At. En. Conf. Vol. 12. pp. 695-704.

Henning, G.R. 1950. The slow neutron absorption cross section of graphite. In: Proc. French-American Conf. Graphite Reactors, ANL-4410.

Jurney, E.T., Motz, H.T. 1963. Thermal neutron capture in $\mathrm{d}$ and ${ }^{16} \mathrm{O}$. In: Int. Conf. Neutron Physics wit Reactor Neutrons, ANL-6797. pp. 236-242.

Jurney, E.T., Bendt, P.J., Browne, J.C., 1982. Thermal neutron capture cross section of deuterium. Physical Review C 25, 2810-2811.
Kikuchi, T., Nagai, Y., Suzuki, T.S., Shima, T., Kii, T., Igashira, M., Mengoni, A., Otsuka, T., 1998. Nonresonant direct p- and d-wave neutron capture by ${ }^{12} \mathrm{C}$. Physical Review C - Nuclear Physics 57 (5), 2724-2730.

Koechlin, J.C., Tanguy, P., Zaletski, C.P. 1957. French results on natural uraniumgraphite lattices, 97-126.

Matsue, H., Yonezawa, C., 2004. The recommended $k_{0}$-factors for neutron-induced prompt gamma-ray analysis and the prompt gamma-ray emission probabilities. Journal of Radioanalytical and Nuclear Chemistry 262 (1) 49-55.

Muehlhause, C.O., Harris, S.P., Rose, D., Schroeder, H.P., Thomas, G.E., Wexler, S., 1957. In: Proc. French-American Conf. Graphite Reactors, BNL-489. p. 19.

S.F. Mughabghab, 2003. Tech. Rep. INDC(NDC)-440, International Atomic Energy Agency Report.

Mughabghab, S.F., Lone, M.A., Robertson, B.C., 1982. Quantitative test of the lanelynn theory of direct radiative capture of thermal neutrons by ${ }^{12} \mathrm{C}$ and ${ }^{13} \mathrm{C}$. Physical Review C 26, 2698-2701.

Nagai, Y., Takeda, K., Motoyama, S., Ohsaki, T., Igashira, M., Mukai, N., Uesawa, F., Ando, T., Kitazawa, H., Fukuda, T., 1991. Neutron capture cross sections of light nuclei in primordial nucleosynthesis. Nuclear Instruments and Methods in Physical Research Section B 56/57, 492.

NEA Nuclear Safety Committee, 2010. No. NEA/NSC/DOC(95)03. OECD/NEA Paris.

Nichols, P.F., 1960. Absorption cross section of graphite. Nuclear Science Engineering 7, 395-399.

Nojiri, N., Shimakawa, S., Fujimoto, N., Goto, M., 2004. Characteristic test of initial HTTR core. Nuclear Engineering and Design 233 (1-3), 283-290.

Ohsaki, T., Nagai, Y., Igashira, M., Shima, T., Takeda, K., Seino, S., Irie, T., 1994. New measurement of the $12 \mathrm{C}(\mathrm{n}, \mathrm{g}) 13 \mathrm{C}$ reaction cross section. Astrophysical Journal $422,912$.

Otuka, N., Dunaeva, S., Dupont, E., Schwerer, O., Blokhin, A., 2011. The role of the nuclear reaction data centres in experimental nuclear data knowledge sharing. Journal of the Korean Physical Society 59 (23), 1292-1297.

Pelowitz Denise B., 2008. MCNPX USERS MANUAL.

Prestwich, W.V., Islam, M.A., Kennett, T.J., 1981. A determination of the carbon thermal-neutron capture cross section. Nuclear Science and Engineering 78, 182

Price, G.A., 1964. Measurement of the transport mean free path of thermal neutrons in graphite by a poison method. Nuclear Science Engineering 18, 410-412.

Rose, P.F., Fu, C.Y., Tech. Rep. ENDF-201: ENDF/B-VI Summary Documentation.

Rosman, K.J.R., Taylor, P.D.P., 1998. Isotopic compositions of the elements 1997. Journal of Physical and Chemical Reference Data 27 (6), 1275-1287.

Sagot, M., Tellier, H., 1963. Mesure des paramètres de diffusion du graphite. Journal of Nuclear Energy A/B 17, 347-348.

Mughabghab, Said, 2006. Atlas of Neutron Resonances. Elsevier (March).

Santamarina, A., Bernard, D., Blaise, P., Coste, M., Courcelle, A., Huynh, T.D., Jouanne, C., Leconte, P., Litaize, O., Mengelle, S., Noguèure, G., Ruggiéri, J.-M., Sérot, O. Tommasi, J., Vaglio, C., Vidal, J.-F., Rugama, Y., 2009. The JEFF-3.1.1 nuclear library. OECD/NEA.

Schillebeeckx, P., Becker, B., Danon, Y, Guber, K., Harada, H., Heyse, J.Junghans, A.R. Kopecky, S., Massimi, C., Moxon, M.C., Otuka, N., Sirakov, I., Volev, K., 2012b. Determination of resonance parameters and their covariances from neutron induced reaction cross section data. Nuclear Data Sheets 113 (12), 3054-3100 (Special Issue on Nuclear Reaction Data).

Shibata, Keiichi, Kawano, Toshihiko, Nakagawa, Tsuneo, Iwamoto, Osamu, Katakura, Jun-Ichi, Fukahori, Tokio, Chiba, Satoshi, Hasegawa, Akira, Murata, Toru, Matsunobu, Hiroyuki, Ohsawa, Takaaki, Nakajima, Yutaka, Yoshida, Tadashi, Zukeran, Atsushi, Kawai, Masayoshi, Baba, Mamoru, Ishikawa, Makoto, Asami, Tetsuo, Watanabe, Takashi, Watanabe, Yukinobu, Igashira, Masayuki, Yamamuro, Nobuhiro, Kitazawa, Hideo, Yamano, Naoki, Takano, Hideki, 2002. Japanese Evaluated Nucl ear Data Li brary Version 3 Revision-3: JENDL-3.3. Journal of Nuclear Science and Technology 39 (11), 1125-1136.

Shibata, Keiichi, Iwamoto, Osamu, Nakagawa, Tsuneo, Iwamoto, Nobuyuki, Ichihara, Akira, Kunieda, Satoshi, Chiba, Satoshi, Furutaka, Kazuyoshi, Otuka, Naohiko, Ohasawa, Takaaki, Murata, Toru, Matsunobu, Hiroyuki, Zukeran, Atsushi, Kamada, So, Katakura, Jun-Ichi, 2011. JENDL-4.0: A New Library for Nuclear Science and Engineering. Journal of Nuclear Science and Technology 48 (1), 130.

Shima, T., Kii, T., Kikuchi, T., Okazaki, F., Kobayashi, T., Baba, T., Nagai, Y., Igashira, M. 1995. Experimental studies of keV energy neutron-induced reactions relevant to astrophysics and nuclear physics. No. 97-004.

Snoj, L., Žerovnik, G., Trkov, A., 2012a. Computational analysis of irradiation facilities at the JSI TRIGA reactor. Applied Radiation and Isotopes 70, 483-488.

Soppera, N., Bossant, M., Dupont, E., Henriksson, H., Rugama, Y., 2011. Recent upgrades to the nuclear data tool JANIS. Journal of the Korean Physical Society 59 (23), 1329-1332

Stankovskiy, A., Van den Eynde, G., 2012. Advanced method for calculations of core burn-up, activation of structural materials, and spallation products accumulation in accelerator-driven systems. Science and Technology of Nuclear Installations.

Starr, E.G., Price, G., 1962. Measurement of the diffusion parameters of graphite and graphite-bismuth by pulsed neutron methods. In: Proc. Brookhaven Conf. Neutron Thermalization, BNL-719. pp. 1034-1073.

Wagemans, J., Vittiglio, G., Malambu, E., Abderrahim, Hamid Ait, 2009. The BR1 reactor: a versatile irradiation facility for fundamental research and industrial applications. In: Advancements in Nuclear Instrumentation Measurement Methods and their Applications (ANIMMA). 